\title{
Rheologic results and their correlation to hemostatic changes in patients with moderate and severe preeclampsia: An observational cross-sectional study
}

\author{
Amr A. Soliman ${ }^{\mathrm{a}, \mathrm{b}, *}$, Roland Csorba ${ }^{\mathrm{a}, \mathrm{c}}$, Asli Yilmaz ${ }^{\mathrm{d}}$ and Georg-Friedrich von Tempelhoff ${ }^{\mathrm{a}}$ \\ ${ }^{a}$ Department of Obstetrics and Gynecology, City Hospital of Aschaffenburg, Teaching Hospital of the \\ Julius, Maximilians University Würzburg, Aschaffenburg, Germany \\ ${ }^{\mathrm{b}}$ Department of Obstetrics and Gynecology, University of Alexandria, Alexandria, Egypt \\ ${ }^{\mathrm{c}}$ Department of Obstetrics and Gynecology, University of Debrecen, Debrecen, Hungary \\ ${ }^{\mathrm{d}}$ Department of Obstetrics and Gynecology, GPR Hospital Rüsselsheim, Rüsselsheim, Germany
}

\begin{abstract}
Previous study have shown an association between failure of physiological hemodilution during 2nd trimester and an increased risk for the development of subsequent pregnancy complications such as early birth, birth of a growth retarded newborn (IUGR), low fetal birth weight and preeclampsia. The latter complication in particular goes along with dramatic changes in the placental perfusion as well as systemic maternal blood flow. Severity of preeclampsia may be preceded by distinct impaired hemodilution and reflected by the results of rheological parameters.

A subgroup analysis was performed in a community based retrospective study of 4,985 consecutively recorded singleton pregnant women of whom 423 had preeclampsia. Mean 2 nd trimester hemoglobin levels and blood rheological results at the time of delivery were assessed and compared in women with moderate and severe preeclampsia. Mean 2nd trimester hemoglobin levels were calculated from the maternal records. Rheological variables included plasma viscosity (KSPV 1 Fresenius) and Red blood cell aggregation in stasis and under low shear conditions (MA1-Aggregometer; Myrenne).

According to the definition of the German Society of Gynecology and Obstetrics (DGGG) 314 women had moderate preeclampsia $(74.2 \%)$, while 109 had severe preeclampsia due to the presence of a blood pressure $>170 / 110 \mathrm{mmHg}(n=41 ; 9.7 \%)$, and/or IUGR $<5$ th percentile $(n=28 ; 6.6 \%)$, and/or HELLP-Syndrome $(n=10 ; 2.4 \%)$, and/or proteinuria $\geq 5 \mathrm{~g} / 24 \mathrm{~h}(n=30 ; 7.1 \%)$. Age, BMI, smoking, and maternal weight were comparable in the groups, while gestational age at delivery as well as fetal outcome parameter were statistically significant unfavourable in patients with severe preeclampsia.

Mean 2nd trimester hemoglobin level were statistically significantly higher in women who developed severe vs. moderate preeclampsia $(\mathrm{m}=12.75 \pm 0.99 \mathrm{~g} / \mathrm{dL}$ vs. $\mathrm{m}=12.50 \pm 1.05 \mathrm{~g} / \mathrm{dL} ; p=0.033)$. However, in the ROC calculations a hemoglobin value of $12.05 \mathrm{~g} / \mathrm{dL}$ revealed best sensitivity (78\%) and specificity $(33.8 \%)$ in women with subsequent diagnosis of severe preeclampsia, whereas sensitivity was $100 \%$ for a value $>10.95 \mathrm{~g} / \mathrm{dL}$. There were no statistically significant differences for none of the rheological parameters at the time of delivery between groups of patient with moderate v.s severe preeclampsia. Severe preeclampsia and IUGR, however, was associated with statistically significantly higher RBC aggregation as compared to patients with moderate preeclampsia. Plasma viscosity was statistically significantly $(p<0.05)$ correlated with Fibrinogen values $(r=1.69)$, leukocyte- $(r=0.11)$ and platelets-count $(r=0.127)$, and hemoglobin/hematocrit values in particular $(r=0.23 / 0.26)$.

Although mean 2 nd trimester hemoglobin concentration are higher in patients with subsequent development of severe preeclampsia, due to the low sensitivity and specificity of this parameter clinical use for identifying women at risk is of limited value. On the other hand, a hemoglobin value below $11.0 \mathrm{~g} / \mathrm{dL}$ excluded the risk for severe preeclampsia to $100 \%$. Blood rheological parameters at the time of delivery in the absence of IUGR are not markedly influenced by severity of preeclampsia. Keywords: Blood fluidity, haemostasis, pregnancy, preeclampsia
\end{abstract}

${ }^{*}$ Corresponding author: Amr A. Soliman, Klinikum Aschaffenburg, Am Hasenkopf 1, 63739 Aschaffenburg, Germany. Tel.: +49 602132 434191; Fax: +49 6024639 0527; E-mail: amr.soliman@klinikum-aschaffenburg.de. 


\section{Introduction}

Hypertensive disorders affect 6 to $8 \%$ of the pregnancies worldwide, and are responsible for 20 to $25 \%$ of maternal mortality [13]. Hypertensive disorders in pregnancy are on top of the maternal killers' list in developed countries but are counted third in developing ones. They are responsible for 50,000 maternal mortalities worldwide annually [31]. Moreover, preeclampsia is responsible for one in every three obstetric morbidities [44] and not only affects maternal outcome, but is also responsible for a five time increase in the risk of a perinatal mortality [31].

A definite cause of preeclampsia could not be identified, despite the obvious impact of this disorder on maternal and fetal outcomes. Many theories and hypotheses were published trying to demonstrate a cause of preeclampsia, but none of them gained enough consensus to be named the sole and only cause of preeclampsia. Among those theories comes the placental ischemia hypothesis [30], the very low-density lipoprotein (VLDL) versus toxicity-preventing activity (TxPA) hypothesis [2, 25], the hyperdynamic disease model [4] and the immune maladaptation hypothesis [10]. Most of the research about preeclampsia was concerned with the microvasculature, pathophysiology of microvasculature control and vascular resistance, which are all related to a vessel wall pathology or disturbance in regulatory mechanisms, although an effect of blood viscosity on the blood flow in the microcirculation is proved to exist $[15,21$, $22,38]$. Blood viscosity is a function of rheological blood parameters like haematocrit, plasma viscosity, RBC aggregability and deformability. Some authors studied the rheological aspect of preeclampsia but these were rather small studies that ran on a limited number of patients, moreover, they either studied hemorheologic or hemostatic changes, but did not correlate them to each other nor to the severity of preeclampsia $[18,19,29]$.

The aim of this work is to quantify the rheological changes at term in patients with moderate vs. severe preeclampsia and to evaluate the prognostic value of hemoglobin concentration during the second trimester in predicting the severety of disease in patients with subsequent development of preeclampsia later on in pregnancy.

This work is actually a supplement to a previously published work that quantified the hemorehological changes in pathological pregnancies in comparison to normal ones in a big cohort of pregnant women [42].

\section{Patients and methods}

All consecutive women who delivered in the obstetric department during the time period from January 1990 to the end of December 1996 were principally eligible to be included into this retrospective investigation. However, blood rheological and hemostaseological estimations were restricted to women with singleton pregnancy from whom a copy of the antenatal care register (Mutterpass) was obtained and whose data were digitalised using a 205 variables protocol. This protocol assessed data from previous pregnancies and deliveries and the current pregnancy. The data included information about risk factors of pregnancy and or delivery, maternal characteristics e.g. routine physical examination, fetal ultrasound documented by the attending Obstetrician, in addition to laboratory test results e.g. hemoglobin-concentrations, proteinuria and immunologic/hematologic results. Moreover, the personal, medical, and social history including risk factors of pregnancy/delivery (gain of weight and systolic/diastolic blood pressure during pregnancy, obesity, smoking, co-morbidity, and drugs) were documented.

Women with preeclampsia (Blood pressure (BP) equal to or more than $140 \mathrm{mmHg}$ systolic or $90 \mathrm{mmHg}$ diastolic in addition to proteinuria of more than $300 \mathrm{mg} / 24 \mathrm{~h}$ or HELLP Syndrome) were recruited from 
the big pool of patients and they were subsequently stratified into moderate preeclampsia or severe preeclampsia. Those with severe preeclampsia were further stratified into four subgroups according to the dominant severity criterion, namely severe hypertension with BP more than or equal to 170 systolic and/or 110 diastolic, fetal growth below 5\% percentile of normal (IUGR: intra uterine growth retardation), severe proteinuria with proteinuria equal or more than $5 \mathrm{~g} / 24 \mathrm{~h}$ and the HELLP syndrome group. This stratification was performed according to the guidelines of the German Society of Obstetrics and Gynecology (DGGG)[11].

\subsection{Rheological parameters}

Estimations of blood rheological parameters were performed at the time of admission into the labor and delivery ward and were repeated after $24 \mathrm{~h}$ until delivery thus calculations are based on the results within a time-range of $24 \mathrm{~h}$ prior to delivery. After minimal stasis of the upper arm blood was drawn from the antecubital vein using a 20 gauge needle supplied with a vacuum tube. Blood was collected in vacuum tubes containing $1: 10$ potassium EDTA (ethylene diamine tetraacetic acid) and rheological estimations were immediately performed in the laboratory of the Department of Gynecology \& Obstetrics according to ICSH guidelines (International Committee for Standardization in Haematology). Hematocrit (packed cell volume) was measured by microcentrifugation (normal range females: 37.0-48.0\%). Red Blood Cell aggregation (RBC aggregation) was estimated using a photometric rheoscope developed by Schmid-Schoenbein et al. [34]. (MA1-Aggregometer; Myrenne, Roetgen, Germany). Blood samples $(20 \mu \mathrm{L})$ adjusted to a standard hematocrit of $45 \%$ were placed between a transparent cone - plate system and rotated for 10 seconds at high shear rate of $600 \mathrm{~s}-1$ in order to disperse all pre-existing cell aggregates. Average RBC aggregation was determined by the quantity of light transmission which is measured by photo sensors in two modes - during stasis - and while samples are subjected to low shear rate of $3 \mathrm{~s}-1$. Light transmission increases proportionally with extend of RBC aggregation. The data are then processed by an integrated computer and expressed in arbitrary units. For determination of plasma viscosity vacuum tubes were centrifuged for 20 minutes $\left(2000 \mathrm{~g}\right.$ at $4{ }^{\circ} \mathrm{C}$ ) whereas probes from the middle-layer of the plasma were obtained and inserted into and measured with the system of a Capillary tube viscosimeter (KSPV 1 Fresenius, Bad Homburg Germany) at $37^{\circ} \mathrm{C}$ according to Jung et al. [23]. (normal range: $1.14-1.34 \mathrm{mPa}$ ).

\subsection{Statistical analysis}

Descriptive analysis included mean values \pm standard deviations, median, inter quartile range and 95\% confidence interval. Differences between groups were assessed with the Wilcox test for unpaired and paired samples and tested against zero. Correlation coefficients according to Spearman were calculated. Two-sided $p$ values of less than 0.05 were considered statistical significant. Statistical analyses were conducted in collaboration with the Institute for Medical Biometry, Epidemiology and Informatics; University of Mainz using SAS 9.0 program package (SAS Institute Berkley CA).

\section{Results}

According to the DGGG (German Society of Obstetrics and Gynecology) definition of preeclampsia [11], 423 pregnant could be identified as having preeclampsia in our cohort. This cohort included 4,985 pregnancies traced in the aforementioned time period. According to the DGGG classification of 
Table 1

Frequency table showing the number and percentage of patients in each of the groups and subgroups of this study

\begin{tabular}{lccc}
\hline & Frequency & Percentage & Cumulative percent \\
\hline Moderate preeclampsia & 314 & 74.2 & 74.2 \\
Blood pressure $>170 / 110 \mathrm{mmHg}$ & 41 & 9.7 & 83.9 \\
Severe IUGR $<$ th percentile) & 28 & 6.6 & 90.5 \\
HELLP syndrome & 10 & 2.4 & 92.9 \\
Proteinuria $\geq 5$ g/24h & 30 & 7.1 & 100 \\
Total & 423 & 100 & \\
\hline
\end{tabular}

hypertensive disorders in pregnancy 314 women have been identified as moderate preeclamptic $(74.2 \%)$, while 109 women (25.8\%) were identified as severe preeclamptic and/or HELLP syndrome. Table 1 shows the frequency distribution of our patients into those with moderate preeclampsia and those with severe preeclampsia which were allocated to subgroups according to their presenting severity criterion. There was no statistically significant difference between the two groups (i.e. moderate and severe preeclampsia) as regards the Age, the BMI, the average daily cigarette consumption and the weight at the beginning of the pregnancy and at delivery as well. The duration of gestation in the severe preeclamptic group, with its subgroups was statistically significantly shorter when compared to the moderate preeclampsia group. The only exception to this observation was the subgroup presenting with severe proteinuria. The magnitude of proteinuria and the values of systolic and diastolic blood pressure at the time of delivery in the severe preeclampsie group were statistically significantly higher compared to the moderate preeclampsia group. The exceptions existed in the HELLP syndrome subgroup as regards proteinuria and the HELLP syndrome and sever proteinuria subgroups as regards systolic/diastolic blood pressure values (Table 2).

\subsection{Mean haemoglobin concentration in the second trimester (14 to 28th gestational weeks) in the pregnant women who developed moderate or severe preeclampsia later in pregnancy}

The mean/median haemoglobin concentration in the second trimester of pregnancy in the patients presenting with moderate and severe preeclampsia was checked among a cohort of 3,184 different haemoglobin concentration values, whereas the median number of times of haemoglobin concentration check during pregnancy for women with moderate preeclampsia was 8 (range $2-20)$ in contrast to 7 (range 2-16) in those who presented with severe preeclampsia $(p=0.28)$ which was the case also in the severe preeclampsia subgroups. The patients presenting with severe preeclampsia had a statistically significant higher haemoglobin concentration in contrast to those presenting with moderate preeclampsia, (mean $=12.75 \pm 0.99 \mathrm{~g} / \mathrm{dL}$; median $12.5 \mathrm{~g} / \mathrm{dL}$ ) and $($ mean $=12.5 \pm 1.05 \mathrm{~g} / \mathrm{dL} ;$ median $=12.4 \mathrm{~g} / \mathrm{dL})$ respectively $(p=0.033)$. In the subgroup analysis the severe hypertensive group (mean $=12.72 \pm 0.99 \mathrm{~g} / \mathrm{dL}$; Median $=12.45 ; p=0.05$ ) and the severe proteinuria group $($ mean $=12.71 \pm 0.84 \mathrm{~g} / \mathrm{dL}$; Median $=13.0 ; p=0.24$ ) showed the highest haemoglobin concentration values (Fig. 1). However, the values were not statistically significant higher than those from the moderate preeclampsia group. The patients in the HELLP syndrome group showed the lowest mean and median haemoglobin concentration values $($ mean $=12.54 \pm 0.71 \mathrm{~g} / \mathrm{dL} ;$ Median $=12.5 ; p=0.75)$.

The ROC curves of the frequency distribution of the hemoglobin values in the second trimester of the 423 confirmed preeclmaptic patients were produced and used to determine the sensitivity and specificity 
Table 2

Comparison of the clinical parameters of the moderate and severe preeclamptic groups at delivery

\begin{tabular}{|c|c|c|c|c|c|c|c|c|c|c|}
\hline & & $N$ & Mean & SD & Median & $95 \%$ & 6-CI & Min & Max & $P$-value* \\
\hline \multirow{6}{*}{$\begin{array}{l}\text { Duration of } \\
\text { pregnancy in } \\
\text { weeks }\end{array}$} & Moderate Preeclampsia & 305 & 38.29 & 2.186 & 39 & 38.04 & 38.53 & 28 & 42 & \\
\hline & Severe Preeclampsia & 105 & 36.54 & 3.107 & 37 & 35.94 & 37.14 & 28 & 41 & 0.0001 \\
\hline & Blood Pressure $\geq 170 / 110 \mathrm{mmHg}$ & 38 & 36.71 & 2.700 & 37 & 35.82 & 37.60 & 29 & 41 & 0.0001 \\
\hline & IUGR & 27 & 34.56 & 3.004 & 35 & 33.37 & 35.74 & 28 & 40 & 0.0001 \\
\hline & HELLP-syndrome & 10 & 35.20 & 4.077 & 36.5 & 32.28 & 38.12 & 28 & 40 & 0.0001 \\
\hline & Proteinuria $\geq 5 \mathrm{~g} / 24 \mathrm{~h}$ & 30 & 38.57 & 1.870 & 39 & 37.87 & 39.26 & 34 & 41 & n.s. \\
\hline \multirow{6}{*}{ Proteinuria (g/24h) } & Moderate Preeclampsia & 305 & 0.91 & 1.464 & 0 & 0.75 & 1.08 & 0 & 15 & \\
\hline & Severe Preeclampsia & 105 & 3.20 & 2.940 & 2.0 & 2.63 & 3.77 & 0 & 9 & 0.0001 \\
\hline & Blood Pressure $\geq 170 / 110 \mathrm{mmHg}$ & 38 & 1.95 & 1.413 & 2.0 & 1.48 & 2.41 & 0 & 5 & 0.0001 \\
\hline & IUGR & 27 & 1.59 & 2.223 & 1.0 & 0.71 & 2.47 & 0 & 9 & 0.029 \\
\hline & HELLP-syndrome & 10 & 1.00 & 1.247 & 0.50 & 0.11 & 1.89 & 0 & 3 & n.s. \\
\hline & Proteinuria $\geq 5 \mathrm{~g} / 24 \mathrm{~h}$ & 30 & 6.97 & 1.650 & 6.0 & 6.35 & 7.58 & 5 & 9 & 0.0001 \\
\hline \multirow{6}{*}{$\begin{array}{l}\text { Duration of } \\
\text { delivery (h) }\end{array}$} & Moderate Preeclampsia & 305 & 4.91 & 4.256 & 4.0 & 4.44 & 5.39 & 1 & 22 & \\
\hline & Severe Preeclampsia & 105 & 2.92 & 3.756 & 1.0 & 2.20 & 3.65 & 1 & 18 & 0.0001 \\
\hline & Blood Pressure $\geq 170 / 110 \mathrm{mmHg}$ & 38 & 3.18 & 4.417 & 1.0 & 1.73 & 4.64 & 1 & 18 & 0.019 \\
\hline & IUGR & 27 & 1.04 & 0.192 & 1.0 & 0.96 & 1.11 & 1 & 2 & 0.0001 \\
\hline & HELLP-syndrome & 10 & 3.00 & 3.266 & 1.0 & 0.66 & 5.34 & 1 & 9 & n.s. \\
\hline & Proteinuria $\geq 5 \mathrm{~g} / 24 \mathrm{~h}$ & 30 & 4.27 & 4.135 & 3.0 & 2.72 & 5.81 & 1 & 14 & n.s. \\
\hline \multirow{6}{*}{$\begin{array}{l}\text { Mean systolic } \\
\text { blood pressure at } \\
\text { delivery }(\mathrm{mmHg})\end{array}$} & Moderate Preeclampsia & 305 & 141.10 & 11.953 & 140 & 139.75 & 142.45 & 100 & 182 & \\
\hline & Severe Preeclampsia & 105 & 158.94 & 20.116 & 160 & 155.05 & 162.84 & 110 & 220 & 0.0001 \\
\hline & Blood Pressure $\geq 170 / 110 \mathrm{mmHg}$ & 38 & 176.39 & 17.157 & 180 & 170.76 & 182.03 & 170 & 220 & 0.0001 \\
\hline & IUGR & 27 & 157.78 & 12.795 & 160 & 152.72 & 162.84 & 135 & 180 & 0.0001 \\
\hline & HELLP-syndrome & 10 & 143.90 & 14.333 & 140 & 133.65 & 154.15 & 120 & 170 & n.s. \\
\hline & Proteinuria $\geq 5 \mathrm{~g} / 24 \mathrm{~h}$ & 30 & 142.90 & 10.978 & 142.5 & 138.80 & 147.00 & 110 & 165 & n.s. \\
\hline \multirow{6}{*}{$\begin{array}{l}\text { Mean diastolic } \\
\text { blood pressure at } \\
\text { delivery }(\mathrm{mmHg})\end{array}$} & Moderate Preeclampsia & 305 & 86.78 & 9.815 & 90 & 85.67 & 87.89 & 59 & 108 & \\
\hline & Severe Preeclampsia & 105 & 98.85 & 13.737 & 100 & 96.19 & 101.51 & 55 & 140 & 0.0001 \\
\hline & Blood Pressure $\geq 170 / 110 \mathrm{mmHg}$ & 38 & 110.34 & 13.346 & 125 & 105.96 & 114.73 & 110 & 140 & 0.0001 \\
\hline & IUGR & 27 & 96.56 & 8.111 & 100 & 93.35 & 99.76 & 69 & 106 & 0.0001 \\
\hline & HELLP-syndrome & 10 & 88.00 & 10.551 & 90 & 80.45 & 95.55 & 70 & 104 & n.s. \\
\hline & Proteinuria $\geq 5 \mathrm{~g} / 24 \mathrm{~h}$ & 30 & 89.97 & 7.467 & 90 & 87.18 & 92.75 & 70 & 100 & n.s. \\
\hline
\end{tabular}

n.s. = non significant.

of hemoglobin values in the second trimester as a parameter to predict preeclampsia. A sensitivity of $100 \%$ was achieved with hemoglobin values $\leq 11 \mathrm{~g} / \mathrm{dL}$ while the specificity was very low, mounting only to less than $7 \%$. The highest possible sensitivity (78\%) and specificity $(33.8 \%)$ were achieved at a hemoglobin value of $12.05 \mathrm{~g} / \mathrm{dL}$. At this point the AUC was 0.56 and lied at the diagonal of the represented area. AUC was 0.55 for severe proteinuria and 0.46 for HELLP syndrome. 


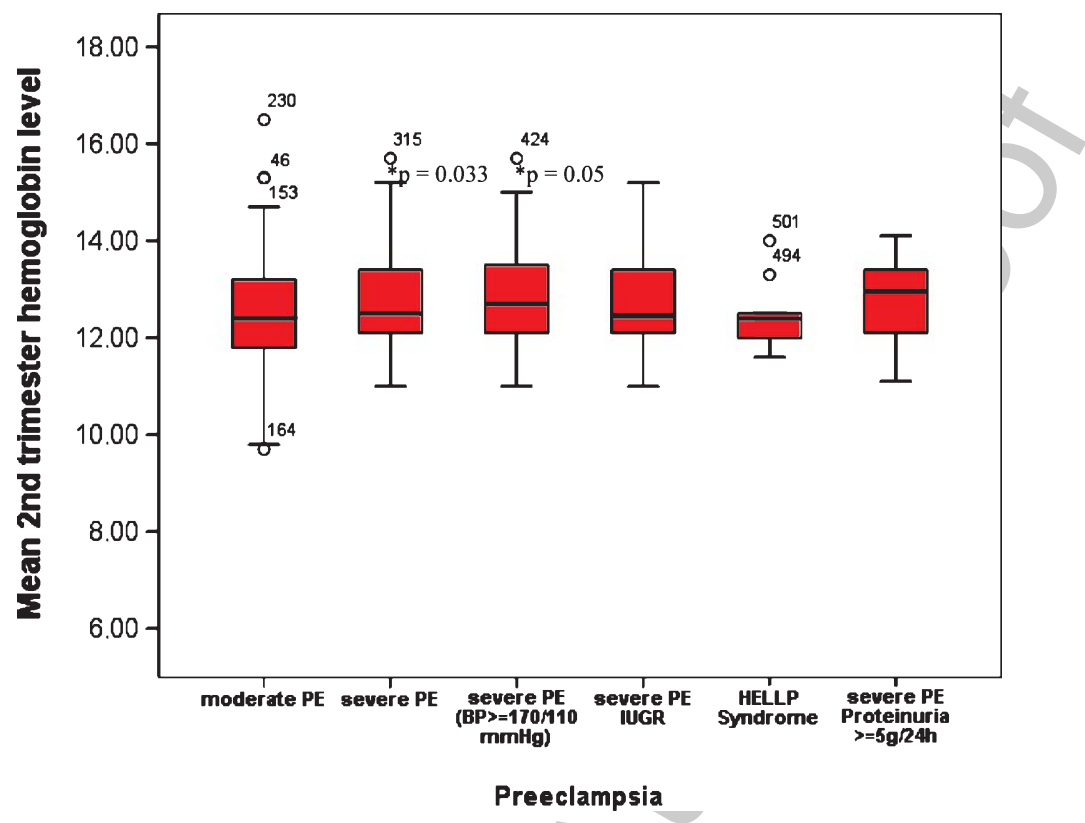

Fig. 1. Box plot of median hemoglobin concentrations in the second trimester of women who will develop preeclampsia later on in pregnancy. (Median, 25 to $75 \%$ interquartiles, minimum and maximum values, outliers). Hemoglobin concentration in $\mathrm{g} / \mathrm{dL}$. ${ }^{*} p$ value statistically significant difference in comparison to the moderate preeclampsia group.

\subsubsection{Changes in hemoglobin concentration between the second trimester estimation and the values taken at term}

The difference between the mean hemoglobin concentration in the second trimester and at term was not statistically significant in the patients with moderate preeclampsia $(\Delta \mathrm{m}=0.05 \pm 1.48 \mathrm{~g} / \mathrm{dL} ; p=0.51)$. On the contrary, the mean hemoglobin concentration in the severe preeclampsia group showed a statistically significant increase of $0.54 \pm 1.71 \mathrm{~g} / \mathrm{dL}(p=0.001)$ between the second trimester and at term. The sub group of severe hypertension in the severe preeclampsia group showed the highest statistically significant surge in hemoglobin concentration $(\Delta \mathrm{m}=0.86 \pm 2.03 \mathrm{~g} / \mathrm{dL} ; p=0.009)$ followed by those patients in the severe proteinuria subgroup $(\Delta \mathrm{m}=0.53 \pm 1.21 \mathrm{~g} / \mathrm{dL} ; p=0.02)$. In contrast to the other subgroups in the severe preeclampsia group, the HELLP syndrome subgroup showed a non statistically significant fall in hemoglobin concentration $(\Delta \mathrm{m}=0.11 \pm 1.58 \mathrm{~g} / \mathrm{dL} ; p=0.83)$. The magnitude of hemoglobin concentration surge between the second trimester and term was statistically significant higher in the severer preeclampsia group in comparison to the moderate preeclampsia group $(p=0.009)$. In the subgroup analysis the hemoglobin concentration surge was statistically significant in the severe hypertension subgroup $(0.017)$ and the severe proteinuria subgroup $(p=0.05)$ in comparison to the moderate preeclampsia group (Fig. 2).

\subsection{Rheological characteristics at delivery of the moderate and severe preeclamptic patients}

The rheological blood tests were done within the 24 hours preceding the delivery. (range 10-1,320 minutes, mean $=575$ minutes). The results of both groups were analyzed i.e. the moderate and the severe preeclampsia and for the subgroups of the severe preeclampsia group. 


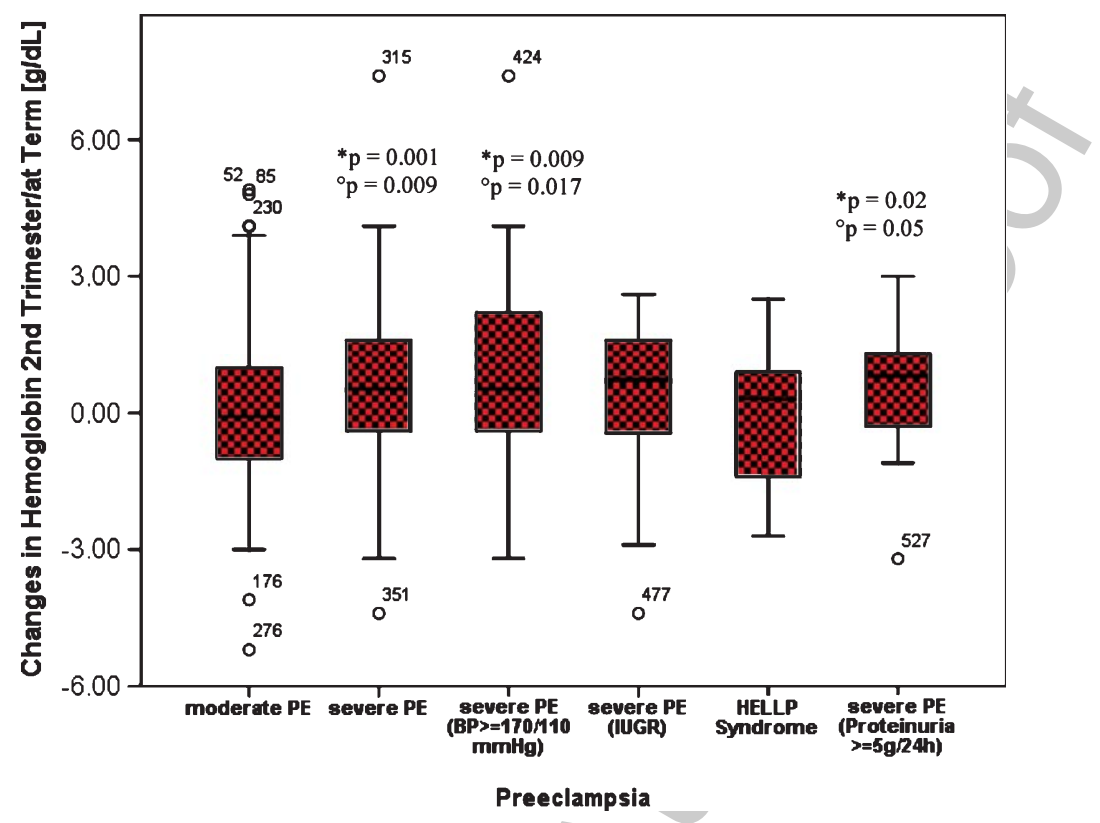

Fig. 2. Box plot of the change in hemoglobin concentrations in the second trimester and at term of women who developed preeclampsia later on in pregnancy. (Median, 25 to 75\% interquartiles, minimum and maximum values, outliers). Difference in hemoglobin concentration in $\mathrm{g} / \mathrm{dL} .{ }^{*} p$ is a statistically significant difference in hemoglobin concentration between the second trimester and the values at term. ${ }^{\circ} p$ is a statistically significant difference in hemoglobin concentration in comparison to the moderate preeclampsia group.

\subsubsection{Haematocrit}

Patients with severe preeclampsia showed a statistically significant lower haematocrit levels $(\mathrm{m}=35.84 \pm 4.48 \%$, median $=36.0 \%, p=0.02)$ in comparison to those with moderate preeclampsia $(\mathrm{m}=36.78 \pm 3.57 \%$, median $=37.0 \%)$. The lowest hemtocrit values in the subgroup analysis was again registered in the severe hypertensive subgroup $(\mathrm{m}=35.0 \pm 4.65 \%$, median $=35.8 \%, p=0.005)$ while those patients in the severe IUGR, severe proteinuria and HELLP syndrome did not show a statistically significant difference from the moderate preeclampsia group. A classical normal distribution of hematocrit values was shown in both preeclampsia groups, where $80 \%$ of the values of the moderate preecplampsia and $60 \%$ of those with severe preeclampsia were between 32 and $41 \%$. The exception was the subgroup presenting with severe proteinuria where the distribution was shifted to the right with $51 \%$ of the values $>38 \%$.

\subsubsection{Erythrocyte aggregation (at stasis)}

A trend of a higher erythrocyte aggregation in the severe preeclampsia group in comparison to the patients with moderate preeclampsia was observed. This did not reach however statistically significance $(\mathrm{m}=23.1 \pm 6.2$; Median $=23.2$; v.s. $\mathrm{m}=22.5 \pm 5.3$; Median $=22.7$, respectively with $p=0.33)$. The only subgroup that showed a statistically significant difference was the subgroup presenting with IUGR in comparison to the moderate preeclamptic group $(m=25.1 \pm 6.5$; Median $=23.9 ; p=0.02)$. The values of this subgroup were also higher when compared to the patients in the severe preeclampsia subgroups. On the contrary, the subgroup with severe proteinuria showed the lowest erythrocyte aggregation values in all subgroups $(m=21.9 \pm 5.8$; Median $=21.6 ; p=0.52)$. The erythrocyte aggregation at stasis was observed 


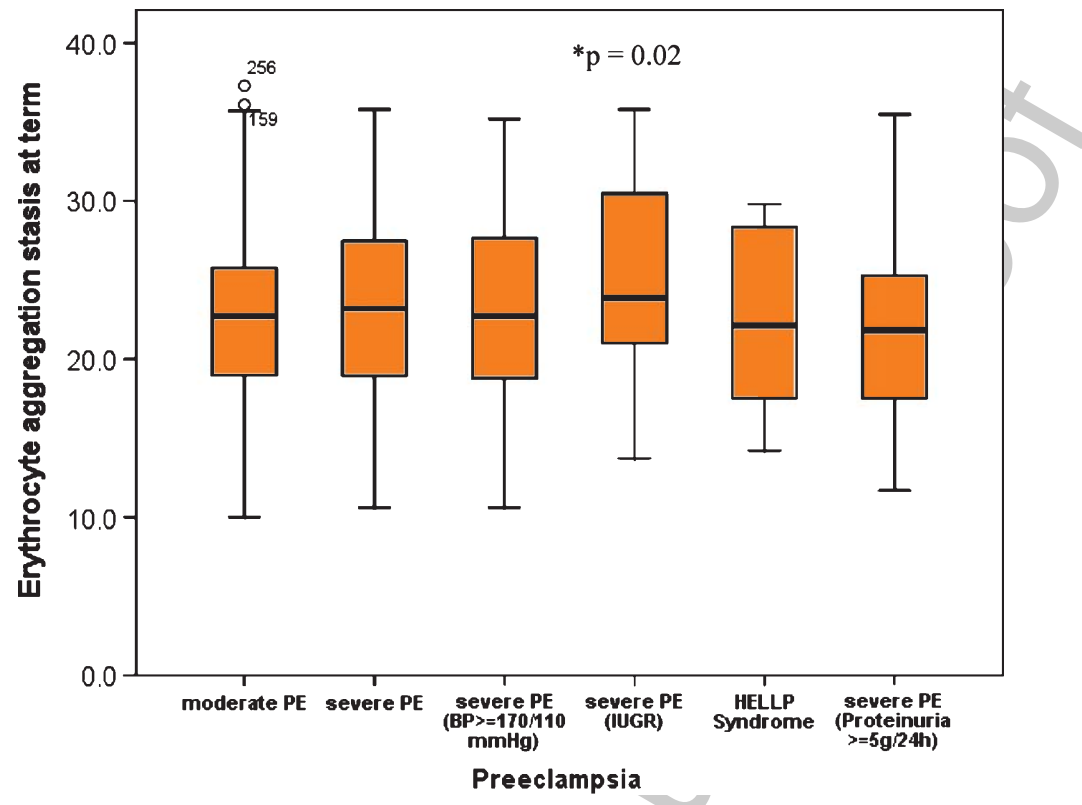

Fig. 3. Box plot of erythrocyte aggregation at stasis at the time of delivery in patients with moderate and severe preeclampsia with its subgroups. (Median, 25-72\% interquartile range, maximum and minimum values and outliers). Erythrocyte aggregation at stasis per second (unit time). ${ }^{*} p$ is a statistically significant value in comparison to the moderate preeclampsia group.

to be in the normal range in both the moderate and the severe preeclampsia groups in $77 \%$ and $73 \%$ of the patients respectively with values between 16.0 and 28.0. Around $23 \%$ of the patients with severe preeclampsia and IUGR had an erythrocyte aggregation in stasis $>31.0$ (Fig. 3).

\subsubsection{Erythrocyte aggregation (under low shear forces)}

The erythrocyte aggregation values of both groups under low shear forces did not show statistically significant difference from each other resembling the values taken at stasis. With a mean of $39.9 \pm 7.93$; Median $=40.3$ in the moderate preeclampsia group and a mean of $41.2 \pm 10.5$; Median $=40.9$ in the severe preeclampsia group a $p$ value of 0.18 could not prove this difference as statistically significant. As it was with erythrocyte aggregation at stasis, the only group of patients that showed a significant difference from the moderate preeclampsia group was those with severe IUGR (mean $=44.8 \pm 11.3$; Median $=43.8 ; p=0.005$ ). Likewise in the erythrocyte aggregation at stasis values, the subgroup with severe proteinuria had the lowest values in relation to the group with moderate preeclampsia but was not statistically significant (mean $=40.3 \pm 8.5$; Median $=39.1 ; p=0.72$ ). The erythrocyte aggregation under low shear forces showed normal values in both groups in $70.3 \%$ and $61 \%$ for the patients with moderate and those with severe preeclampsia respectively where the values ranged between 32 and 47. The maximum fluctuation in the erythrocyte aggregation values under low shear forces was found among the subgroup with HELLP syndrome whereas a fifth of the patients with IUGR had a value over 57 (Fig. 4).

\subsubsection{Plasma viscosity}

The plasma viscosity showed no statistically significant difference among both groups of preeclampsia with a mean of $1.32 \pm 0.09 \mathrm{mPas}$; Median of $1.32 \mathrm{mPas}$ in the moderate preeclampsia group and a mean 


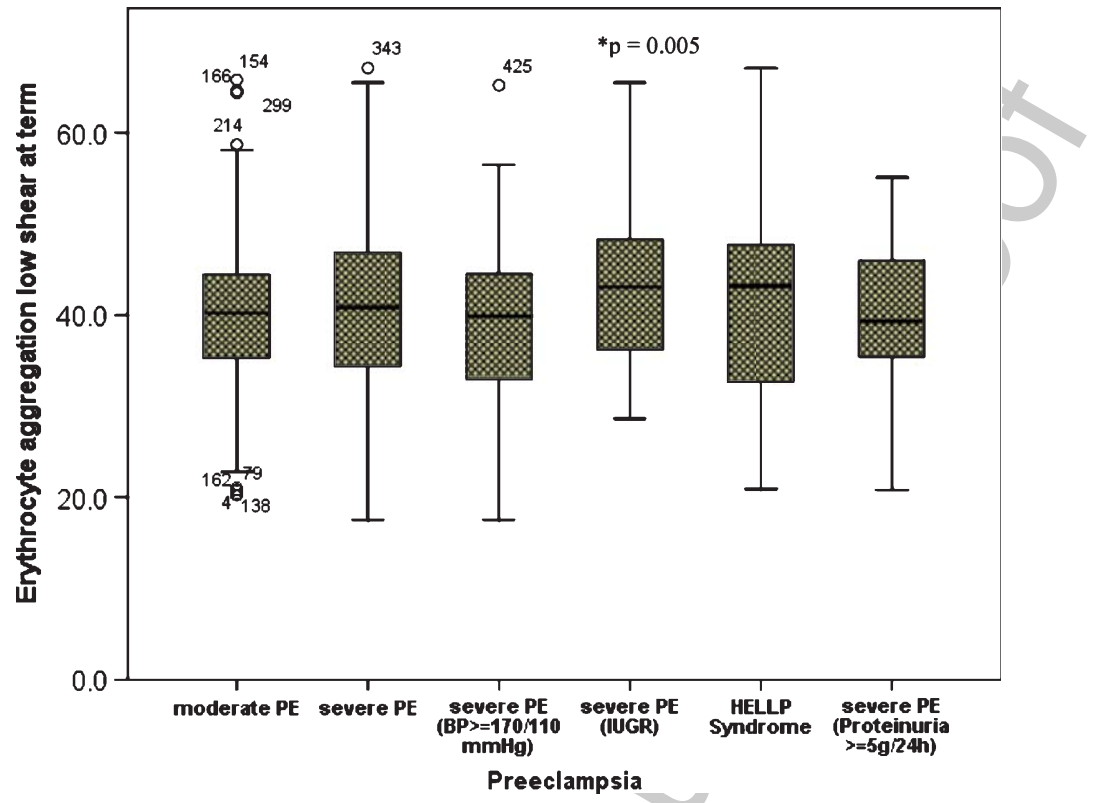

Fig. 4. Box plot of erythrocyte aggregation under low shear forces at the time of delivery in patients with moderate and severe preeclampsia with its subgroups. (Median, 25-72\% interquartile range, maximum and minimum values and outliers). Erythrocyte aggregation under low shear forces at term per second (unit time). ${ }^{*} p$ is a statistically significant value in comparison to the moderate preeclampsia group.

of $1.31 \pm 0.09$; Median of $1.32 \mathrm{mPas}$ in the severe preeclampsia group $(p=0.21)$. This non significant difference was also seen when comparing the subgroups of the severe preeclampsia patients whose plasma viscosity values ranged between 1.31 and $1.33 \mathrm{mPas}$. The only exception could be observed in the HELLP syndrome subgroup of patients whose mean plasma viscosity was $1.23 \pm 0.1 \mathrm{mPas}$ with a Median of $1.22 \mathrm{mPas}$, the values that were statistically significant different from those in the moderate group of preeclampsia with a $p$ value of 0.01 (Fig. 5).

$81.3 \%$ and $85.1 \%$ of the patients in the moderate and those in the severe preeclampsia groups respectively had plasma viscosity values between 1.2 and $1.45 \mathrm{mPas}$. In the subgroups of the severe preeclampsia patients, the values ranged between 1.35 and $1.4 \mathrm{mPas}$ (34\% of those with severe proteinura and $29 \%$ of those with severe IUGR). A left hand shift was seen in patients with HELLP syndrome where $50 \%$ of the values lied below $1.25 \mathrm{mPas}$.

\subsubsection{Correlation the rheological and the hemostaseological parameters and standard tests at delivery}

A relationship could be found between the different, above mentioned, rheological test results on one side and coagulation profiles and hemostase standard tests on the other side. The coagulation profile of the patients was tested at the same time where the rheological tests were done when the patient was admitted for delivery. A significant correlation could not be found between the activated Partial Thromboplastin Time (aPTT) values and any of the rheological parameters that were tested. On the contrary, plasma viscosity was found to be inversely proportional to the (Prothrombin Time) PT values $(r=-0.137 ; p=0.006)$. Plasma viscosity showed a weak statistically significant positive correlation to anti-thrombin activity $(r=0.11 ; p=0.027)$ and showed a positive correlation to high plasma fibrinogen 


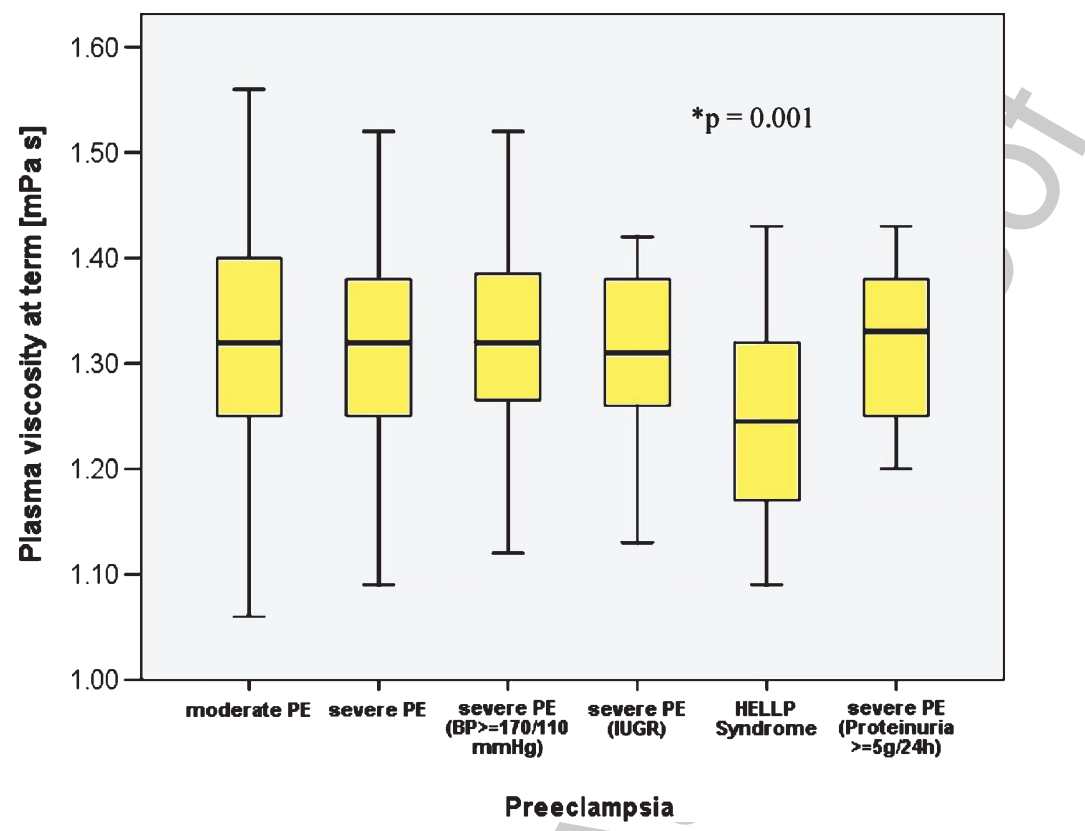

Fig. 5. Box plot of plasma viscosity at the time of delivery in patients with moderate and severe preeclampsia with its subgroups. (Median, 25-72\% interquartile range, maximum and minimum values and outliers). Plasma viscosity in mPa. ${ }^{*} p$ is a statistically significant value in comparison to the moderate preeclampsia group.

concentrations $(r=0.169 ; p=0.001)$. In this correlation pattern, a significant correlation could be found between increasing erythrocyte aggregation values both at stasis and under low shear forces with high fibrinogen concentrations $(r=0.115 ; p=0.023$ and $r=0.171 ; p=0.001$, respectively).

The different components of the blood corpuscular count showed a significant positive correlation with plasma viscosity at delivery. For the leucocytes count $(r=0.11 ; p=0.02)$, for the thrombocytes $(r=0.127 ; p=0.008)$, for the haematocrit $(r=0.26 ; p<0.001)$ and for haemoglobin concentration $(r=0.238 ; p<0.001)$. The single case where the correlation was found to be statistically significant negative (inversely proportional) was between erythrocyte aggregation at stasis and thrombocytic count $(p=0.02)$.

\section{Discussion}

Hemorheologic changes in pregnancies complicated with preeclampsia were investigated in a few studies [21, 29], that tried to enhance our understanding of the changes that occur in this disorder whose etiology is still unexplained. Preeclampsia remains one of the unsolved maternal health problems worldwide. The results of our study will be added to the existing studies in literature tackling this point, which might help in understanding the pathophysiological mechanisms of preeclampsia.

Our data showed a paradox for hematocrit values. Hematocrit values normally fall in pregnancy [5] but in case of preeclampsia, hematocrit values variably increase according to the severity of hemoconcentration to which the patient is subjected [36] and are found to be at significantly higher than normal pregnancies at term [42]. In our cohort the significantly lower hematocrit values in the severe preeclampsia group in comparison to the moderate group is a real paradox. It could not be explained by low corpuscular 
count because the hemoglobin values were not significantly lower in the severe preeclampsia group, and even though, in the HELLP syndrome group, which, by definition exhibits hemolysis and consequently a fall in red corpuscular count, did not show significantly lower hematocrit values neither in the subgroup analysis nor in comparison to the moderate group. Moreover, the observation that the severe hypertension group had the lowest hematocrit values among all groups adds a lot of questions to this paradox. Cirillo and the Gubbio study research group proved through their work on 2,809 hypertensive men and women that prevalence of hypertension was at least two times greater for persons whose hematocrit levels were higher by 10 units [8]. Our observations might be contradicting to their findings because our population was only composed of pregnant women.

Plasma viscosity values in pregnancy were always controversial, with some studies reporting no change with gestation [12, 16], some reporting an increase [20, 37], some a decrease [17], and others an initial increase followed either by no significant changes [14] or by a subsequent decrease [7]. In case of preeclampsia, the reports are not in a better harmony, with reports showing hyperviscosity [6], others showing no relevant changes [18]. Our results conform to a great extent with what Heilmann et al. published. He compared a small cohort of severe preeclamptic women to women bearing normal pregnancies and could not find a significant higher plasma viscosity levels in preeclamptic women [18]. In our cohort a significantly higher plasma viscosity could not be proved to exist when comparing severe to moderate preeclampsia group, where the only difference in this aspect between our work and Heilmann's study, apart from the cohort size, is that he compared the preeclamptic women to normal pregnant women whereas in our study the comparison was with moderate preeclamptic women. The only group in our cohort that showed significantly higher plasma viscosity than the moderate group was those patients presenting with HELLP syndrome. We did a literature database search on Pubmed.org with the key words Hemolysis AND Plasma viscosity with the hope to find an explanation to this observation, but our efforts were in vain. We could not find a publication that related or studied the connection of hemolysis to plasma viscosity. Our possible explanation to these results is the release of various protein particles from the destructed RBCs that could add more to the protein load of the blood plasma and hence increase the viscosity in addition to the elevated fibrinogen turnover in our patients. The latter explanation could be supported by our observation of a moderate positive correlation between plasma viscosity and fibrinogen concentration. We tried also to correlate plasma viscosity changes to the known Doppler flow changes in preeclampsia. Von Tempelhoff and his colleague were able to find evidence that correlates plasma viscosity to Doppler flow changes through their small scale randomized study. They proved that hemodilution was significantly related to improvement in Doppler flow parameters both on the maternal side (uterine artery Doppler indices) and on the fetal side (fetal aorta Doppler indices). Moreover, they related the better improvement in the fetal Doppler flow indices in one of their two groups in comparison to the second group to the Hydroxyethyl-starch (HES) initiated reduction in plasma viscosity and erythrocyte aggregation inhibition [40]. Connes et al. studied the plasma viscosity and hemodynamic changes but in exercise. They found an increase in plasma viscosity during exercise that was inversely proportional to systemic vascular resistance. They found a significant positive correlation between nitric oxide production and subsequent vasodilatation with plasma viscosity. They used both findings to suggest that decreased vascular resistance during exercise was related to a plasma viscosity induced increase in endothelial nitric oxide production [9]. Nitric oxide levels in preeclampsia are controversial, some authors suggest an increase [3, 43] others found a decrease [33] and some reported an unchanged nitric oxide level [28]. The correlation between plasma viscosity and nitric oxide might explain how plasma viscosity and hemodynamics interact in preecalampsia and raises the question if rheologic changes correlate to Doppler flow changes in preeclampsia. 
When it concerns erythrocyte aggregation, our data could not show a significantly higher erythrocyte aggregation in the severe preeclampsia group when compared to the moderate group - just a higher trend was observed. The only exception was the IUGR subgroup that had significantly higher erythrocyte aggregation when compared to the moderate preeclampsia group. This data applied to erythrocyte aggregation both at stasis and under low shear forces. Heilmann et al. found a significantly higher erythrocyte aggregation in severe preeclampsia both at stasis and under low shear forces [29] which is conform with our findings, although in the IUGR subgroup still needs to be explained. During our literature search we could only identify one study that precisely investigated this point. Voigt and his colleague found a significantly higher erythrocyte aggregation in cases presenting with fetal growth restriction at term. Those, whoever, were not necessarily due to preeclampsia [39]. Michalska-Maleska and her colleague found an increased erythrocyte aggregation in patients with primary open angle glaucoma among other rheological changes and incriminated those changes to decreased blood flow to the optic nerve with the subsequent development of neuropathy [26]. We believe a similar mechanism could relate increased erythrocyte aggregation to IUGR, where the blood flow resistance could increase in the spiral arterioles pouring into the placental bed on the maternal side due to this event, causing the fetal hypoperfusion and the fetal growth retardation.

Our results found a weak positive correlation between plasma viscosity and anti-thrombin activity, an inversely proportional relationship between aPTT and plasma viscosity, a positive correlation between plasma viscosity and fibrinogen, a positive correlation between plasma viscosity and blood corpuscular components count and thrombocytic count was inversely proportional to erythrocyte aggregation at stasis. Physiological fibrinogen increase in pregnancy contributes to the increase in plasma viscosity [5] and our results are in accordance with this finding. Prolonged aPTT reflects activated coagulation process and hence a consumption of coagulation factors, which include fibrinogen. So it is a consequence of the finding that increasing fibrinogen levels contribute to increasing plasma viscosity that a decrease in fibrinogen, signaled by a prolonged aPTT, will correlate to lowered plasma viscosity. The anti-thrombin is a small molecular weight protein [35] and the likelihood that its increase will contribute to increase in plasma viscosity is low. It is not clear the reason of the positive correlation between plasma viscosity and the various blood corpuscular components counts. Neither is it understandable this negative correlation between the thrombocytic count and the erythrocyte aggregation at stasis.

Haemoglobin concentrations in the second trimester in our cohort were significantly higher among those presenting with severe preeclampsia than those presenting with moderate preeclampsia. This is contradicting the findings of Ali et al. who concluded through their study on 9,578 deliveries that maternal anemia is a significant risk factor for developing preeclampsia [1], but was conforming with the findings of other researchers that states that excess maternal serum iron [24] or excess maternal hemoglobin is related to the development of preeclampsia later in pregnancy [27, 32, 41]. Moreover, we used the mean hemoglobin values in the second trimester to anticipate the development of preeclampsia later on in the pregnancy. In our previous study the mean second trimester hemoglobin concentration was a significant predictor for the development of preeclampsia later on in pregnancy [29], the finding which was reconfirmed with this work. However, a differentiation of the severity of preeclampsia using hemoglobin concentration is associated with a low sensitivity/specificity. Interestingly, anemia of less than $11 \mathrm{~g} / \mathrm{dL}$ at the second trimester excluded the development of severe preeclampsia. These findings are very encouraging to integrate hemoglobin concentration in the second trimester into a screening model with uterine artery Doppler in the process of early anticipation of preeclampsia.

Our findings concerning the changes in hemoglobin concentration between the second trimester and term in moderate and severe preeclampsia are non-preceded, especially that the significant rise in 
hemoglobin concentration in the severe preeclampsia group between second trimester and term in comparison to the moderate preeclampsia group is contra acted by a significantly lower hematocrit value in the severe peeclampsia group at term which is interesting enough to be further validated and investigated.

\section{Acknowledgments}

The authors declare no conflict of interest related to this manuscript.

\section{References}

[1] A.A. Ali, D.A. Rayis, T.M. Abdallah, M.I. Elbashir and I. Adam, Severe anaemia is associated with a higher risk for preeclampsia and poor perinatal outcomes in Kassala hospital, eastern Sudan, BMC Research Notes 4 (2011), 311-316.

[2] B.W. Arbogast, S.C. Leeper, R.D. Merrick, K.E. Olive and R.N. Taylor, Which plasma factors bring about disturbance of endothelial function in preeclampsia? Lancet 343 (1994), 340-341.

[3] S. Bhatnagar, J. Bhattacharjee, J.M. Vaid, T. Madan, S.S. Trivedi and P.U. Sarma, Inducible nitric oxide synthase (iNOS) gene polymorphism in preeclampsia: A pilot study in North India, Aust N Z J Obstet Gynaecol 47 (2007), 477-482.

[4] P.M. Bosio, P.J. McKenna, R. Conroy and R. O’Herlihy, Maternal central hemodynamics in hypertensive disorders of pregnancy, Obstet Gynecol 94 (1999), 978-984.

[5] F. Broughton-Pipkin, Maternal Physiology In: Dewhurst's Textbook of Obstetrics and Gynaecology D. Keith Edmonds Ed. Blackwell Publishing 2007, (7th edition) 10-18.

[6] P.C. Buchan, Preeclampsia - a hyperviscosity syndrome, Am J Obstet Gynecol 142 (1982), 111-112.

[7] P.C. Buchan and H.N. MacDonald, Rheological factors in Obstetrics and Gynaecology. In: G.D.O. Lowe, J.C. Barbenel, C.D. Forbes Eds. Clinical Aspects of Blood Viscosity and Cell Deformity. Springer Verlag, Berlin, Heidelberg, New York, 1981, 465-478.

[8] M. Cirillo, M. Laurenzi, M. Trevisan and J. Stamler, Hematocrit, Blood pressure and Hypertension. The Gubbio Population Study, Hypertension 20 (1992), 319-326.

[9] P. Connes, A. Pichon, M.D. Hardy-Dessources, X. Waltz, Y. Lamarre, M.J. Simmonds and J. Tripette, Blood viscosity and hemodynamics during exercise, Clin Hemorheol Microcirc 51 (2011), 101-109.

[10] G.A. Dekker and B.M. Sibai, Etiology and Pathogenesis of preeclampsia: Current Concepts, Am J Obstet Gynecol 179 (1998), 1359-1375.

[11] Deutsche Gesellschaft für Gynäkologie und Geburtshilfe (DGGG), Arbeitsgemeinschaft Schwangerschaftshochdruck/Gestose Diagnostik und Therapie hypertensiver Schwangerschaftserkrankungen. AWMF 2010 015/018 (S2):1-24.

[12] L. Dintenfass, Haemorheological changes in pregnancy, Contrib Nephrol 25 (1981), 91-97.

[13] L. Duley, Maternal mortality associated with hypertensive disorders of pregnancy in Africa, Latin America and the Caribbean, Br J Obstet Gynaecol 99 (1992), 547-553.

[14] R.D. Eastham, Plasma viscosity estimation in the improved haematological screening of antenatal patients, J Obstet Gynaecol Br Cmnwlth 72 (1965), 763-764.

[15] T. Gori, A. Damaske, S. Muxel, M.C. Radmacher, F. Fasola, S. Schaefer, M. Fineschi, S. Forconi, F. Jung, T. Münzel and J.D. Parker, Endothelial function and hemorheological parameters modulate coronary blood flow in patients without significant coronary artery disease, Clin Hemorheol Microcirc (2012), DOI: 10.3233/CH-2012-1603.

[16] H. Hamilton, Blood viscosity in pregnancy, J Obstet Gynaecol Br Cmnwlth 57 (1950), 530-538.

[17] L. Heilmann, Blood rheology and pregnancy, Baillieres Clin Haematol 1 (1987), 777-799.

[18] L. Heilmann, W. Rath and K. Pollow, Hemorheological changes in women with severe preeclampsia, Clin Hemorheol Microcirc 31 (2004), 49-58.

[19] L. Heilmann, W. Rath and K. Pollow, Hemostatic abnormalities in patients with severe preeclampsia, Clin Appl Thromb Hemost 13 (2007), 285-291.

[20] T.C. Inglis, J. Stuart, A.J. George and A.J. Davies, Haemostatic and rheological changes in normal pregnancy and preeclampsia, Br J Haematol 50 (1982), 461-465. 
[21] H.S. Ji and S.J. Lee, In vitro hemorheological study on the hematocrit effect of human blood flow in a microtube, Clin Hemorheol Microcirc 40 (2008), 19-30.

[22] F. Jung, C. Mrowietz, B. Hiebl, R.P. Franke, G. Pindur and R. Sternitzky, Influence of rheological parameters on the velocity of erythrocytes passing nailfold capillaries in humans, Clin Hemorheol Microcirc 48 (2011), 129-139.

[23] F. Jung, H.G. Roggenkamp, R. Schneider and H. Kiesewetter, The capillary tube plasma viscosimeter. A new apparatus for measuring plasma viscosity, Biomed Tech (Berl) 28 (1983), 249-252.

[24] T.T. Lao, K.-F. Tam and L.Y. Chan, Third trimester iron status and pregnancy outcome in non-anaemic women; pregnancy unfavourably affected by maternal iron excess, Hum Reproduct 15 (2000), 1843-1848.

[25] B. Lerentzen, M.J. Endresen, T. Clausen and T. Henriksen, Fasting serum free fatty acids and triglycerides are increased before 20 weeks of gestation in women who later develop preeclampsia, Hypertens Pregnancy 13 (1994), 103-109.

[26] K. Michalska-Malecka and L. Slowinska-Lozynska, Aggregation and deformability of erythrocytes in primary open-angle glaucoma (POAG); the assessment of arterial hypertension, Clin Hemorheol Microcirc 51 (2012), 277-285.

[27] J.F. Murphy, J. O'Riordan, R.G. Newcombe, E.C. Coles and J.F. Pearson, Relation of hemoglobin levels in first and second trimesters to outcome of pregnancy, Lancet 1 (1986), 992-995.

[28] S.J. Orange, D. Painter, J. Horvath, B. Yu, R. Trent and A. Hennessy, Placental endothelial nitric oxide synthase localization and expression in normal human pregnancy and preeclampsia, Clin Exp Pharmacol P 30 (2003), 376-381.

[29] D.J. Pepple, M.R. Hardeman, A.M. Mullings and H.L. Reid, Erythrocyte deformability and erythrocyte aggregation in preeclampsia, Clin Hemorheol Microcirc 24 (2001), 43-48.

[30] C.W.G. Redman, G.P. Sacks and I.L. Sargant, Preeclampsia: An excessive maternal inflammatory response to pregnancy, Am J Obset Gynecol 180 (1999), 499-506.

[31] J.M. Roberts, Pregnancy related hypertension. In: RK Creasy, R Resnik (eds). Maternal Fetal Medicine. 4th ed. W.B. Saunders, Philadelphia, 1998, 833-872.

[32] S.P. Salas, G. Marshall, B.L. Gutierrez and P. Rosso, Time course of maternal plasma volume and hormonal changes in women with preeclampsia or fetal growth restriction, Hypertension 47 (2006), 203-208.

[33] V.C. Sandrim, A.C. Palei, I.F. Metzger, V.A. Gomes, R.C. Cavalli and J.E. Tanus-Santos, Nitric oxide formation is inversely related to serum levels of antiangiogenic factors soluble fms-like tyrosine kinase-1 and soluble endogline in preeclampsia, Hypertension 52 (2008), 402-407.

[34] H. Schmid-Schönbein, J. von Gosen, L. Heinich, H.J. Klose and E. Volger, A counter-rotating 'rheoscope chamber' for the study of the microrheology of blood cell aggregation by microscopic observation and microphotometry, Microvasc Res 6 (1973), 366-376.

[35] W.H. Seegers, J.F. Johnson and C. Fell, An antithrombin reaction to prothrombin activation, Am J Physiol 176 (1954), 97-103.

[36] A. Shennan, Hypertensive disorders. In: Dewhurst's Textbook of Obstetrics and Gynaecology D. Keith Edmonds Ed. Blackwell Publishing 2007 (7th edition) 227-235.

[37] J. Thorburn, M.M. Drummond and K.A. Whigham, Blood viscosity and haemostatic factors in late pregnancy, preeclampsia and fetal growth retardation, Br J Obstetr Gynaecol 89 (1982), 117-122.

[38] I.A. Tikhomirova, A.O. Oslyakova and S.G. Mikhailova, Microcirculation and blood rheology in patients with cerebrovascular disorders, Clin Hemorheol Microcirc 49 (2011), 295-305.

[39] T. Voigt and H. Voigt, Rheologic studies in intrauterine retardation and pregnancy-induced hypertension, Zentralbl Gynakol 109 (1987), 1053-1058.

[40] G.F. von Tempelhoff and L. Heilmann, The effect of plasma volume expansion on the utero-placental perfusion, Clin Hemorheol 19 (1993), 729-736.

[41] G.-F. von Tempelhoff, L. Heilmann, L. Rudig, K. Pollow, G. Hommel and J. Koscielny, Mean Maternal Second-trimester Hemoglobin Concentration and Outcome of Pregnancy: A Population-Based Study, Clin Appl Thromb Hemost 14 (2008), 19.

[42] G.F. von Tempelhoff, E. Velten, A. Yilmaz, G. Hommel, L. Heilmann and J. Koscielny, Blood rheology at term in normal pregnancy and in patients with adverse outcome events, Clin Hemorheol Microcirc 42 (2009), 127-139.

[43] P. Vural, Nitric oxide/endothelin-1 in preeclampsia, Clinica Chimica Acta 317 (2002), 65-70.

[44] M. Waterstone, S. Bewley and C. Wolfe, Incidence and predictors of severe obstetric morbidity. Case-control study, $B M J$ 322 (2001), 1089-1094. 